\title{
Connessioni. Intrecci di testi, parole e storie, per ascoltare l'altro e sé stessi
}

\section{Connections. Linking texts, words and stories, to listen to each other and to yourself}

\author{
Daniele Dell'Agnola \\ SUPSI, Dipartimento formazione e apprendimento \\ $\square$ daniele.dellagnola@supsi.ch
}

Riassunto / Nel contributo si descrive un lavoro svolto con allievi tredicenni che hanno cercato di esplorare, ricreare e ricercare i significati del proprio vissuto attraverso il testo letterario e attraverso la scrittura. La relazione tra i giovani lettori e ciò che era loro familiare ha rappresentato il motore di un percorso didattico mosso da una continua conversazione tra l'insegnante, le allieve e gli allievi e i testi. L'itinerario si è rivelato essere un'occasione per riflettere sulle connessioni possibili tra il testo letterario e chi, adolescente, svolge dei compiti di lettura e scrittura.

Parole chiave: leggere; scrivere; significati impliciti; conversazione; letteratura per ragazzi.

Abstract / The contribution describes the work carried out with thirteen-year-old students who have tried to explore, recreate, and research the meaning of their own experience through literary texts and through writing. The relationship between young readers and what was familiar to them represented the driving force behind the didactic path driven by a constant conversation involving the teacher, the students, and the texts. The teaching program turned out to be an opportunity to reflect on the possible connections between the literary text and those who carry out reading and writing tasks, namely teenagers.

Keywords: reading; writing; implicit meanings; conversation; youth literature.

\footnotetext{
(C) 2021 Daniele Dell'Agnola. Questo è un articolo Open Access, sottoposto a un processo di revisione tra pari a doppio cieco, pubblicato dal Centro competenze didattica dell'italiano lingua di scolarizzazione e dal Servizio risorse didattiche e scientifiche, eventi e comunicazione del DFA-SUPSI in collaborazione con l'Alta scuola pedagogica dei Grigioni. L'articolo è distribuito sotto i termini della Licenza Creative Commons Attribuzione - Non commerciale - Non opere derivate 4.0 Internazionale (CC BY-NC-ND 4.0).
} 


\section{Connessioni}

Lo studioso, accademico inglese Richard Hoggart ha scritto che la letteratura “esplora, ricrea, ricerca i significati dell'esperienza umana”. Attraverso la letteratura

gli uomini guardano alla vita con tutta la vulnerabilità, l'onestà e la profondità che è loro concessa e sperimentano le proprie visioni attraverso una relazione del tutto unica con la forma e con il linguaggio.

(Hoggart, 1970, citato da Chambers, 2011, p.16)

Leggere testi letterari permette di capire meglio (o scoprire) chi siamo attraverso la relazione che, in qualità di lettori, intratteniamo con l'opera e i suoi significati: è ben difficile leggere e comprendere senza provare una qualche reazione, noia compresa. Nell'introduzione a Siamo quello che leggiamo saggio dello scrittore per ragazzi Aidan Chambers (2011), Domenico Barrilà sostiene che

non può essere posta alcuna cesura tra chi scrive, ciò che è scritto e colui che legge, così l'azione che chiamiamo lettura diventa un'interazione di mondi dagli esiti fortemente probabilistici. La distinzione tra soggetto e oggetto quasi scompare e l'esito di tale interazione diventa ogni volta imprevedibile.

(Chambers, 2011, p. 16)

La letteratura (la letteratura per ragazzi non è da meno) favorisce la crescita interiore, lo sviluppo emotivo, sfida le nostre convinzioni, ci stimola a cercare nuove soluzioni ai problemi della vita, consente di sperimentare le possibilità, i pericoli senza subirne direttamente le conseguenze.

Connettersi con la realtà presente, con il passato, anche nella scrittura di un racconto, individuare elementi del proprio vissuto nella storia letta significa stimolare l'empatia e lo spirito critico di cui abbiamo bisogno, come umani, per crescere. I processi che favoriscono la lettura profonda, cioè quella che permette al cervello di attivare le dimensioni affettive, richiedono più tempo (perché i processi sono più sofisticati). Nel suo libro Lettore, vieni a casa Maryanne Wolf (2020) scrive di quanto sia necessario questo lavoro di attivazione neuronale. 
Si pensi a come può riverberarsi nei piccoli lettori la scena in cui Pinocchio prende il suo babbo per mano, risale su per la gola del pesce cane, scavalca tre filari di denti e, prima di fare il gran salto nel mare per salvarsi, invita Geppetto a montare a cavalluccio sulle sue spalle, abbracciandolo forte forte. Il figlio che porta il padre alla salvezza, il filo teso tra i due personaggi che si scioglie nella salvezza dell'abbraccio e nel tuffo nel mare, tranquillo come l'olio.

Trovare il me stesso, il mio mondo nel libro, non è sufficiente alla comprensione di un'opera letteraria, ma è un primo passaggio utile a favorire l'interesse, la gratificazione, il piacere per la lettura. Gli insegnanti hanno il compito di aiutare nella scelta dei testi, di orientare allieve e allievi a coglierne i significati più immediati (attraverso la discussione) ma anche di portare le/i giovani a una consapevolezza più profonda sulle qualità del testo, sulle sue strutture, a un livello di lettura in cui la storia personale sia distinta dall'opera in quanto tale. Maggiori sono le occasioni di indagine, maggiori saranno le conoscenze e le possibilità di collegamenti anche tra i testi: si tratta di una competenza che gratifica e incoraggia a scoprire gradualmente altra letteratura, proiettandosi verso la complessità.

Per riflettere sulle connessioni possibili tra il testo e chi, adolescente, svolge dei compiti di lettura e scrittura, presento un incontro con allievi tredicenni che hanno cercato, almeno in parte, di esplorare, ricreare e ricercare i significati della loro esperienza attraverso il testo letterario e attraverso la scrittura di un racconto. La relazione tra i giovani lettori e ciò che era loro familiare ha rappresentato il motore che ha mosso queste iniziative attraverso le quali l'ascolto era mosso da una continua conversazione tra l'adulto, l'allievo e il testo.

\section{Connettersi leggendo}

\subsection{Succede tutto}

Nel suo racconto intitolato La prima volta che ho detto no, tratto dal volume La prima volta che, Beatrice Masini (2016) narra di un ragazzo di nome Pablo che un sabato sera, pur essendo solo in casa, decide di non uscire per verificare se Chiara, la sua amata, si accorge della sua assenza e del vuoto che lascia. Il protagonista, che non si è mai dichiarato per non esporsi alla possibile sconfitta, sviluppa una teoria dello spazio: quando le persone mancano (sono assenti o sono morte) si crea un vuoto, come una bolla che si gonfia e occupa spazio. 
Uno che di solito era lì, c'era sempre e poi sparisce, cosa lascia? Quella specie di buco che occupava si restringe e si annulla, viene schiacciato dalla presenza degli altri, o resta lì, come se fosse il suo posto, ad aspettare che torni e lo occupi di nuovo? [...] secondo la mia teoria dello spazio, per tornare al principio, se non ci sei e crei un vuoto da qualche parte vuol dire che ne stai riempiendo uno da un'altra. Quindi c’è un equilibrio nell'esserci e non esserci. Vale anche per i morti, credo, in un modo un po' diverso. Tipo il nonno: non c'è ma c'è, non è più qui ma tutte le volte che lo penso riprende la sua forma, si fa largo tra le cose di tutti i giorni e c'è, e come se c'è! Mi piace immaginarli così, i morti, come palloni che di solito stanno lì a mezz'aria un po' svuotati e si gonfiano quando qualcuno li ricorda, solo che sono trasparenti, come ci immaginiamo i fantasmi, e meno male, altrimenti il mondo sarebbe pieno di sagome che dondolano a qualche centimetro da terra, come fanno i palloni veri quando cominciano a sfiatare l'elio, e noi quando camminiamo dovremmo farci largo tra loro, leggeri ma ingombranti, i morti nostri e anche quelli degli altri, un bosco, una foresta intera di palloni mezzi sgonfi da scostare con le mani.

(Masini, 2016, pp. 39-40)

Il racconto si chiude con Chiara, accortasi dell'assenza di Pablo. È lei che suona alla porta:

Sabato sera che è adesso non sono uscito per la prima volta da quando ho cominciato a uscire

e non è un gioco di parole è la verità

e ho fatto bene

ho corso un rischio e fatto una cosa pericolosa

e ho fatto bene

perché adesso

adesso

adesso

adesso è domenica e sta succedendo tutto.

(Masini, 2016, p. 49)

Le ultime due parole del racconto ci permettono di lavorare su due livelli di comprensione: l'autrice ha voluto concludere con il soggetto posposto «tutto». La scelta ci porta a riflettere sull'uso del pronome indefinito nel finale aperto della storia. Ho chiesto agli allievi di strutturare una recensione del racconto 
spiegando questi concetti maturati nella conversazione in classe (Allegato 1). Ma c'è molto di più: per capire cosa contiene il «tutto», le allieve e gli allievi hanno dovuto connettersi con il proprio immaginario fatto di desideri, aspettative, futuro, senso dell'amore. Ho assegnato il compito di raccontare in poche righe cosa accade dentro a quel «tutto» integrando olfatto, vista, tatto. Una sfida linguistica ed empatica preparata nella discussione in classe e in una fase di lavoro individuale: nella solitudine si impara ad avere fiducia nell'immaginazione. Un allievo definisce così quel momento indefinito:

(1)

Allora l'ho presa per le anche e l'ho portata a me. La sua pelle era come il pelo di un gatto. Eravamo vicini e lei mi ha baciato.

Accanto alla recensione, così vincolata, ho quindi concesso un momento di scrittura libero, più intimo, su un foglio personale, da conservare, ma non da condividere in classe. Non riporto perciò altri estratti di questa esperienza.

Leggendo la storia ci siamo soffermati sul coraggio di Pablo, disconnesso per una sera: vive la solitudine e rischia di non essere notato; nel contempo alimenta il desiderio, l'attesa che qualcosa accada. E leggere questo racconto in un momento in cui ci si osserva protetti dalle mascherine acquista un significato particolare. Pablo vive la vergogna, si immerge nel sentimento di vulnerabilità, intravvediamo la possibilità del rifiuto che molti adolescenti conoscono. Nel vis à vis conclusivo c'è un "non detto" da colmare, ed è proprio il lettore che lo deve fare, sostando con profondità per riuscire a verbalizzare il proprio immaginario, prima di scrivere la scena.

Il libro contiene anche altre storie attorno alle quali le allieve e gli allievi hanno lavorato, individuando i passaggi nei quali potessero riconoscere una propria esperienza emotiva. Cito ad esempio La prima volta che ci ho provato di Zita Dazzi (2016), dove a provarci non è il protagonista maschile, ma la ragazza, Uma Lampone, abile calciatrice con due caviglie a forma di bullone, ginocchia geometriche, «ginocchia di qualcuno che non ha paura di lanciarsi a terra» (p. 184), appassionata lettrice della Gazzetta dello sport. Nel gioco dei sensi (vista, udito, olfatto, tatto) c'è un climax che porta il protagonista a scoprire il primo 
bacio, in un capovolgimento dei cliché molto interessante per discutere in classe il ruolo di genere. «Mi ero chiesto subito se con una così ci avrei potuto provare» (p. 184) dice il ragazzo che si sente imbranato. Ma alla fine

era come se improvvisamente fosse venuto a galla un altro me che non conoscevo e che stranamente aderiva e si incastrava perfettamente con la ragazza che avevo davanti. Come se improvvisamente non fossi più quello imbranato che avevo sempre sospettato di essere. Come se fossi davvero io, quello che Uma stava baciando.

(Dazzi, 2016, p. 194)

\section{2 lo e la storia}

Per indagare le connessioni tra le lettrici, i lettori e le storie, ho proposto in classe il racconto L'amore ti sposta la testa (Dell'Agnola, 2016) contenuto nel libro dedicato all'educazione sessuale e all'affettività, destinato agli allievi della scuola media ticinese e intitolato L'incontro. In un lavoro prima individuale orientato dall'insegnante, in seguito discusso in piccoli gruppi, allieve e allievi hanno strutturato un taccuino degli appunti suddiviso in quattro sezioni (Allegato 2):

- cosa è successo;

- frasi tratte dal testo;

- la copertina del testo;

- io/noi e il testo.

Ecco la storia in estrema sintesi, riassunta da tre allievi che hanno rivisto insieme le proprie sintesi elaborate precedentemente:

(2)

Questo testo inizia con la presentazione di Alice (la protagonista), la sua famiglia e i suoi compagni di classe. In sintesi: Alice ama Max, ma poi bacia Andrea. Marina ama Andrea ma poi ha un rapporto sessuale con un ragazzo più grande ed infine Max ama Jessica ma lei si fidanza con Nathan. Da non dimenticare, la professoressa Müller e il prof. Kurt che si sposano e hanno un figlio: Ettore. Questo ci fa pensare a delle relazioni strane perché ognuno ricorda un bacio, una cotta o una delusione. Questo alimenta ancora di più il cambiamento, perché grazie alle speranze i ragazzi crescono e maturano. 
Le ragazze e i ragazzi hanno in seguito individuato frasi secondo loro rilevanti, motivandone la scelta (Allegato 2, Allegato 3): «la fecondazione è una pioggia di vita sopra un prato accogliente»; «l'amore è una roba che ti sposta il cervello da un'altra parte»; «praticamente è stato come mettere le ali»; «due robe che sembrano di due mondi lontani e diversi stanno in tensione e poi boom, si mettono insieme e fanno l'amore»; «lascia andare le cose e fai quello che ti senti»; «la vita non è mai una linea diritta». Il compito ha richiesto un dialogo nei gruppi di lavoro, in modo che le allieve e gli allievi potessero essere autonomi nel rintracciare, in un terzo momento, le scene del racconto, le emozioni, le sensazioni nelle quali riconoscersi. C’è chi è rimasto toccato dalla scena finale, in cui Nathan, pur essendo timido e riservato, si dichiara ad Alice lasciandole un bigliettino dove ha scritto il titolo di un racconto di Primo Levi (Erano fatti per stare insieme). Alcuni sostengono di non ritrovarsi in nessun personaggio, ammirando però il coraggio di Nathan (Allegato 2), altri hanno messo in evidenza le urla della madre di Alice, il fatto di voler fare nuove esperienze, "prendere in giro i maestri» e «andare verso il mondo degli adulti» (Allegato 3). Riporto un testo che presenta delle incertezze nelle formulazioni, in seguito discusse e sistemate in classe:

\section{(3)}

Noi adolescenti siamo accomunati alla storia da più cose. Il rapporto tra Alice e Andrea è esemplare, i due si attraggono ma forse non è vero amore e c'è una certa confusione. I protagonisti però crescono fisicamente, nel modo di pensare e nel modo di vivere i sentimenti. Marina e Alice litigano per amore ma tornano a parlarsi dimostrando una grande maturità. Nei cambiamenti mentali si intende Alice che guardando sempre Max non si rende conto di provare qualcosa per Andrea. Anche Marina cambia, prima ama Andrea e con Alice parla solo di lui, poi si scopre che ha fatto l'amore con un diciassettenne. Ha vissuto quindi un'esperienza che l'ha segnata, messa alla prova, anche questo ci accomuna al testo.

Le copertine alternative al volume, disegnate dalle/dai tredicenni, sono pure significative. Abbiamo il caos dei sentimenti e il bigliettino di Nathan che si dichiara citando un racconto di Primo Levi (Allegato 2), l'orologio della vita (Allegato 3), e i due mondi opposti: il risveglio dei sentimenti e i conflitti (Allegato 4). 
Il fatto che questi compiti siano maturati prima individualmente con l'aiuto dell'insegnante, poi in un momento di condivisione, ha aiutato allieve e allievi a concedersi un attimo di respiro per connettersi alla storia e alla propria interiorità, indagando le risposte possibili.

\section{Connessioni degli scrittori}

\subsection{Trasformare la rabbia}

Le allieve e gli allievi devono poter esitare, dubitare e cercare quando leggono, ma anche quando hanno l'occasione di pensare alla scelta di una parola che sia adeguata per esprimere un concetto: una sfida verso la profondità. Con la stessa classe ho letto la Rimetta d'amore furioso di Bruno Tognolini (2011):

Mare in burrasca, terra in tempesta

Se non mi ami, ti spacco la testa

(Tognolini, 2011, p. 34)

Il ritmo, la rima tempesta - testa, la struttura 3+3 nel primo verso e 4+4 nel secondo, gli elementi della natura e quelli degli affetti e dei conflitti costituiscono un'impalcatura interessante, una traccia, un modello per connettere i lettori a «frammenti di vita interiore», come suggerisce Chiara Carminati (2011, pp. 105107) nel suo saggio dedicato al viaggio nelle stanze della poesia con i bambini e i ragazzi. Ecco in che modo abbiamo lavorato (Allegato 5): dopo aver discusso e analizzato la rima in classe, con varie digressioni sulle emozioni che l'amore evoca e sul rispetto dell'altro («se ami qualcuno, non è impossibile che gli spacchi la testa»; «sì, ma queste cose succedono... che un uomo diventa violento») ho chiesto alle allieve e agli allievi di immaginare di riscrivere questi due versi dedicandoli a una persona amata (la mamma, il papà, una compagna, un compagno) senza indicarne il nome. Il testo sarebbe diventato una Rimetta d'amore per te. Come diventa il mare, in questo nuovo testo? E la terra? E se scegliessimo un altro elemento della natura? Potremmo sostituire il mare e la terra con il cielo, le foglie, gli uccelli, l'erba, il lago, l'acqua, il fiume. Cosa dirò all'amata/o? Parte così una sfida linguistica e di connessione all'interiorità molto importante, perché il ragazzo che ha scritto «Mare tranquillo, terra fer- 
ma / tu sei come una lanterna» discutendo la scelta della parola «lanterna» in modo consapevole (la rima ferma - lanterna è molto bella) sta formulando un pensiero profondo. Dichiarerà, nella lettura ad alta voce delle rime, di aver dedicato il testo alla madre. C'è chi invece ha mantenuto il segreto della dedica (Allegato 6). I testi prodotti sono stati illustrati. Ho avuto l'impressione di vivere un momento potente tra parole e voci connesse con l'interiorità e con l'altro:

(4)

Arriva dal bosco un vento lontano

Ti guardo da vicino e ti prendo per mano

\subsection{Fotografie}

Molte poesie d'amore erano riferite alla mamma, come se questi allievi sentissero il bisogno di accostarsi al nido; una sorta di nostalgia dell'infanzia, pur proiettata verso il futuro. Allora, per chiudere l'esperienza di connessione, ho mostrato un videoracconto illustrato tratto dall'opera Flotsam di David Wiesner (2006), disponibile anche online in questo video: https://www.youtube.com/ watch?v=3MTKWnxzqvM\&t=93s.

Il protagonista trascorre il tempo sulla spiaggia osservando i dettagli con la lente, interessato a scoprire universi microscopici. I genitori sono sullo sfondo. La scena mi ricorda un passaggio tratto dal libro di Sherry Turkle (2016, p. 94) sulla conversazione necessaria: nel capitolo «Solitudine» la Turkle parla della noia, «stimolo potente» che «incendia la fantasia e costruisce le risorse emotive interiore». La capacità di giocare da solo del bambino protagonista è centrale: improvvisamente viene travolto da un'onda che lascia sulla spiaggia un apparecchio fotografico. Il ragazzo toglie il rullino dalla macchina e si rivolge al negozio dove si sviluppano le fotografie (storie d'altri tempi che vanno spiegate agli allievi di oggi). Scopre così scatti di mondi meravigliosi e soprattutto l'immagine di un ragazzo che tiene tra le mani una foto di una bambina a sua volta immortalata con una foto di un bambino, eccetera, in una matrioska che, grazie al microscopio, ci porta indietro nel tempo. 
Lo stimolo iniziale mi è servito per chiedere alle allieve e agli allievi di cercare negli album di famiglia una fotografia attorno alla quale avremmo lavorato insieme. L'indagine ha restituito materiale interessante, così ognuno ha potuto indagare il passato della nonna, del nonno, della prozia, del bisnonno. Accanto alle immagini abbiamo annotato le azioni, i pensieri possibili, le emozioni di quel momento preciso: c'era la possibilità di inventare, ma anche di intervistare dei conoscenti che potessero aiutare a ricostruire le esperienze di vita della persona immortalata nella fotografia, che andava setacciata al microscopio, con pazienza.

Ho in seguito assegnato il compito: il personaggio della foto scrive una lettera al futuro. Racconta di sé, di quel momento e proietta dei desideri.

Caro te, nel futuro, chiunque tu sia,

mi trovo qui e penso che

Le consulenze del docente nella raccolta delle idee, nell'indagine, nella formulazione delle domande e soprattutto nel disegno della struttura testuale sono state essenziali e molto importanti. Abbiamo dedicato, in classe, due ore per lo studio dei dati raccolti, quattro ore per la scrittura e la revisione dei testi, due ore per la condivisione. È stato fondamentale chiedere alle allieve e agli allievi, durante le consulenze individuali, di raccontami la storia del personaggio oralmente (Cosa avrebbe scritto nella lettera?). Ho sempre risposto evidenziando gli aspetti interessanti del testo, ma anche quelli non chiari. In questi momenti cardine del laboratorio ho proposto il gioco dei se: «Cosa succederebbe se nel testo tu inserissi questo oggetto, questa sequenza, questa immagine del reale che diventa simbolica? Cosa succederebbe se tu tagliassi questa parte? Il racconto sarebbe coerente? Ascolta il risultato possibile senza questo passaggio.»

In T1 (Allegato 7) l'allievo sceglie l'immagine di sé a due anni. Abbiamo allora modificato il compito. È lui che scrive a se stesso nel passato, evocando un piccolo incidente a causa del quale si era procurato un taglio profondo. L'allievo si porta ancora una cicatrice. Abbiamo allora giocato sull'immagine reale della cicatrice e sull'idea che nella vita ognuno si porta dentro delle cicatrici, delle piccole ferite. In T2 l'allieva ha presentato la fotografia di sua madre che 
a 5 anni stava nell'auto decapottabile del fratello ventenne. Abbiamo sviluppato l'idea della libertà, della partenza dal paese associandola all'automobile e all'indipendenza. In T3 la fotografia del bambino che gioca con il pallone in una strada sterrata in Sardegna ha offerto l'occasione per costruire una scena in cui il pallone (gioco dell'infanzia) ad un certo punto rotola sulla strada e se ne va. È il segnale, per il nonno dell'allieva, che bisogna partire, emigrare in Svizzera. Così i testi hanno acquistato maggiore spessore.

\section{Conclusione}

Sherry Turkle (2016, p. 95) racconta di un insegnante di recitazione in difficoltà nella preparazione di una recita scolastica:

Gli dico che recitare non significa performance verbale, anzi in realtà, quello che l'attore deve davvero fare è ascoltare nel profondo, per rispondere agli altri attori. Eppure gli studenti non riuscivano a stare fermi abbastanza a lungo per ascoltare l'altro. Alla fine l'insegnante di recitazione diede loro un ultimatum: ascoltare attentamente oppure abbandonare la recita. L'ultimatum ebbe i suoi effetti: un gruppo di studenti si ritirò dalla rappresentazione.

(Turkle, 2016, p. 95)

Mi è accaduto il contrario in un laboratorio di creazione teatrale destinato a future/i insegnanti della scuola elementare della SUPSI/DFA, nel momento in cui dieci di loro hanno scelto di raccontare il Covid vissuto dal punto di vista degli studenti. Per svolgere questo compito ho chiesto loro di cogliere l'occasione per leggere in profondità le emozioni, dedicando tempo all'interiorità per scrivere dei brevi monologhi che avrebbero trasferito, trasformato anche nei contenuti, cucito su personaggi da mettere in scena e da interpretare, con le intenzioni giuste. Abbiamo collocato al centro del chiostro della scuola la sedia della solitudine: il respiro dell'interiorità è servito, con qualche aiuto da parte del formatore, per vivere il momento della rappresentazione, della condivisione, insieme a tutti i 50 compagni del corso. Lo spettacolo durava dodici minuti durante i quali accadeva ciò che di umano può succedere nella crisi: i personaggi ballavano in una dimensione spensierata di festa, accompagnati da 
un ritmo travolgente. Improvvisamente un uomo corpulento con un megafono urlava che la festa era finita. Allora tutti si svestivano e trascinavano gli indumenti colorati ai lati del chiostro prima di appendere i vestiti a un cappio. In quel momento si dava spazio al vissuto attraverso i monologhi e poi al grido di rabbia straziante, collettivo. Il lavoro si chiudeva con un abbraccio e una riapertura con dieci sguardi rivolti al futuro, pieni di speranza. Nell'Allegato 8 si vede la sedia sulla quale hanno interpretato quei monologhi di venti, trenta secondi l'uno, in quattro minuti molto intensi, di ascolto e connessione. Ecco alcune tracce di quei testi, che mi preme citare in questo sguardo conclusivo:

(5)

Vi posso dire quello che penso? Io penso che non andrà tutto bene, ed è questo che mi spaventa. La mia paura più grande? E quella che mio figlio non possa vivere la sua infanzia.

(6)

Caro diario, ho 18 anni e due bambini. Tra poco ho gli esami e sono sola. I miei nonni hanno bisogno di me appunto i miei figli hanno bisogno di me. Sono sola. Spero di essere abbastanza forte.

(7)

Facevo delle cene spettacolari con dieci, venti, a volte trenta persone. Ora non si può più. Ma i miei amici? Dove sono finiti? (Cambio posizione sulla sedia) Non ci sono più. Mi hanno abbandonato.

Il Covid? Un sacco di possibilità. Io in giro per le vette delle nostre montagne dalla mattina alla sera. Che spettacolo!

Mia nonna non esce dalla sua stanza da tre anni. Guarda la tv tutto il giorno. Realtà e finzione sono la stessa cosa. Lei viaggia con la sua mente. (Cambio posizione sulla sedia) In questo momento è più libera di noi.

(10)

Sapete cosa vi dico? Qui non ce la raccontano giusta, perché voi pensate di essere liberi, ma in realtà siete delle pedine. (Mi giro, me ne vado, poi torno con l'indice puntato contro il pubblico) Delle pedine! 
(11)

Desidero allontanarmi dalle conversazioni e dalle persone. Vorrei poter mettere un muro tra me e gli altri. Io, una stanza vuota e nessuno con cui dover comunicare. (Breve pausa) È questo ciò che mi serve.

La mia ipotesi è che la scuola debba occuparsi dell'essenziale, che è fatto di complessità. Nell'epoca della connessione elettronica, in cui vi è pure una forte esigenza di misurare gli apprendimenti a costo di sottoporre prove che siano comparabili grazie alla loro neutralità culturale, dobbiamo essere vigili nei confronti dei bisogni educativi delle/dei giovani: se sono allenati a connettersi con i testi, le parole, le storie, se sono allenati ad ascoltare l'altro e se stessi, hanno molte più probabilità di sviluppare le capacità empatiche e la delicatezza di pensiero che li renderà cittadini pronti al dialogo, a costruire la critica senza prevaricare. Diamo il tempo all'essenziale.

\section{Bibliografia}

Carminati, C. (2011). Perlaparola. Bambini e ragazzi nelle stanze della poesia. Modena: Equilibri.

Chambers, A. (2011). Siamo quello che leggiamo. Modena: Equilibri.

Dazzi, Z. (2016). La prima volta che ci ho provato. In AA. VV., La prima volta che (pp. 182-194). Milano: Il Castoro.

Dell'Agnola, D. (2016). L'amore ti sposta la testa. In AA. VV., L'incontro. Testo per gli allievi della scuola media dedicato ai temi della sessualità e dell'affettività (pp. 9-92). Bellinzona: DECS. http://www.scuoladecs.ti.ch/incontro

Hoggart, R. (1970). Speaking to Each Other. London: Chatto \& Windus.

Masini, B. (2016). La prima volta che ho detto no. In AA. VV., La prima volta che (pp. 33-49). Milano: Il Castoro.

Tognolini, B. (2011). Rime di rabbia. Milano: Salani. 
Turkle, S. (2016). La conversazione necessaria. La forza del dialogo nell'era digitale. Torino: Einaudi.

Wiesner, D. (2006). Flotsam. Boston: Houghton Mifflin Harcourt. https://www. youtube.com/watch?v=3MTKWnxzqvM\&t=93s

Wolf, M. (2020). Lettore, vieni a casa. Il cervello che legge in un mondo digitale. Milano: Vita e pensiero. 